\title{
CAlCulated Versus MeAsured URine OSMOLARITY: ACCURACY OF ESTIMATED URINE DENSITY
}

\author{
José de Jesús Vidal-Mayo ${ }^{1}$, Antonio Olivas-MartíneZ ${ }^{1}$, IVÁn PéRez-DíaZ ${ }^{1,2 *}$, JuAn M. LóPez-NAVARro ${ }^{1}$, \\ Elizabet Sánchez-Landa ${ }^{2}$, Eduardo Carrillo-Maravilla ${ }^{1}$, Martha Portillo-González ${ }^{1}$, Reynerio

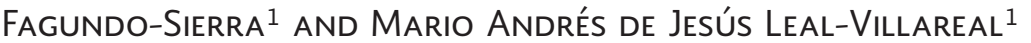 \\ ${ }^{1}$ Instituto Nacional de Ciencias Médicas y Nutrición Salvador Zubirán; ${ }^{2}$ Tecnológico de Monterrey. Mexico City, México
}

\begin{abstract}
Background: Urine osmolarity (OsmU) is the gold standard for the evaluation of the kidney's urine concentration capacity; nevertheless, urinary density (UD) is often used as a surrogate for its estimation. Objective: The objective of this study was to analyze the accuracy of UD in estimating OsmU. Materials and Methods: A transversal study including patients with simultaneous determination of UD measured with refractometry and OsmU measured by osmometer (OsmUm). We multiplied the last two digits of the UD by $35,30,32,33.5$, and 40 to estimate OsmU; the estimates were considered precise if the value was $\pm 30 \mathrm{mOsm} / \mathrm{kg}$ from the OsmUm. A Bland-Altman analysis was conducted. Results: Among 205 patients, there was no difference between OsmUm and the estimated form when using a factor of $33.5(p=0.578)$. When analyzing by the absence or presence of proteinuria and/or glycosuria, there were no differences when using the factors $35(p=0.844)$ and 32 with adjusted UD ( $p=0.898)$. In the linear correlation analysis, values for Pearson's $r=0.788$ and $r^{2}=0.621$ were obtained $(p<$ 0.001 ). The areas under the curve obtained by the receiver operating characteristics curves to estimate urine osmolarity values $<100$ and > $600 \mathrm{mOsm} / \mathrm{kg}$ were $>0.90$. Conclusion: The estimation of the OsmU from UD showed adequate performance. If an osmometer is unavailable, we recommend using the factor 35 for clean samples and 32 with adjusted UD for samples with proteinuria and/or glycosuria. (REV INVEST CLIN. 2018;70:310-8)
\end{abstract}

Key words: Urinary osmolarity. Urinary density. Renal function. Hyponatremia.

Corresponding author:

*Iván Pérez-Díaz,

Instituto Nacional de Ciencias Médicas

y Nutrición Salvador Zubirán

Vasco de Quiroga, 15

Col. Belisario Domínguez Sección XVI, Del. Tlalpan $\quad$ Received for publication: 21-05-2018

C.P. 14080, Mexico City, Mexico

E-mail: ivan.endocrino@gmail.com

Approved for publication: 28-07-2018 doi: $10.24875 / R I C .18002598$ 


\section{INTRODUCTION}

Urinary osmolarity (OsmU) is considered the gold standard in evaluating renal urinary concentration capacity. It is a common tool for clinical practice in scenarios such as assessment of acute kidney injury (AKI), chronic kidney disease (CKD), polyuria, and hyponatremia ${ }^{1-3}$. In cases with hyponatremia with suppressed antidiuretic hormone (ADH) activity, OsmU is usually $<100 \mathrm{mOsm} / \mathrm{kg}^{2}$, while in cases of hyponatremia presenting with $\mathrm{ADH}$ activity, OsmU generally exceeds the plasmatic osmolarity ${ }^{4}$. It is accepted that there is discreet $\mathrm{ADH}$ activity when OsmU $>100 \mathrm{mOsm} / \mathrm{kg}^{5,6}$.

Direct measurement by osmometer is currently the gold standard for osmolarity determination in clinical practice ${ }^{1}$. However, OsmU determination by osmometer is not available at most institutions; thus, urinary density (UD) is used for the indirect calculation of OsmU. It is obtained by multiplying the last two digits of the UD by 35 (multiple of 35,000 times [UD-1] or increase of $35 \mathrm{mOsm}$ per 0.001 increase in the UD) $)^{2,7}$. This method has not been validated, and some factors may alter the determination of OsmU using UD such as age, sex, race, body mass index, CKD, total protein consumption, and water intake ${ }^{8}$. Factors associated with a diluted urine sample include female sex, advanced age, ethnicity, and greater water intake $^{8}$. Due to these limitations, some studies have argued against the utility of UD as a surrogate for OsmU, while clearly favoring the direct determination with osmometry as a tool for evaluating urinary concentration ${ }^{9,10}$.

The aim of this study is to analyze the accuracy of the OsmU calculation when using the UD (OsmUc) compared to direct measurement of OsmU (OsmUm). Another aim is to evaluate the formulas' performance for discrimination of the specific OsmU cutoff points most often used (100 and $600 \mathrm{mOsm} / \mathrm{kg}$ ) in the clinical setting.

\section{MATERIALS AND METHODS}

This was a transversal, comparative, and retrolective study conducted from September 2015 to January 2017 at an academic third-level hospital in Mexico City. We included patients from the emergency department, hospitalization, and outpatient clinics, who underwent simultaneous measurement of Os$\mathrm{mUm}$ and urinalysis (UA) to obtain the UD. The exclusion criteria were patients in hydric restriction protocol, with $\mathrm{AKI}$ in the context of CKD, a urinary tract infection diagnosis, and incomplete laboratory results for analysis or without results corresponding to the same day of the urine sample. The institutional review board approved the study, and informed consent was waived (approval number 2480).

UD, which represents the relationship between the density of a given urine volume against the density of the same volume of distilled water at a constant temperature, was measured by refractometry ${ }^{1}=$ OsmUm was determined by an osmometer (advanced MicroOsmometer Model 3320, Advanced Instruments, Inc.) that uses the freezing point method for osmolarity determination.

OsmU (OsmUc) was indirectly estimated by multiplying the last two digits of the UD by 35 (35,000 times [UD-1] or increase of $35 \mathrm{mOsm}$ per increase of 0.001 in the UD) ${ }^{2,11}$. Osmolarity was also estimated using UD with the factors $30,32,33.5$, and $40^{1,12}$.

From the UA, the following variables capable of influencing the estimation of UD were selected: urine $\mathrm{pH}$, urine protein, urine hemoglobin, urine glucose, urine bilirubin, and ketones. The classifications of the analyzed variables as quantitative and qualitative are shown in table S1. The UD results were also adjusted for proteinuria and glycosuria influence (Formulas S1 and S2).

To evaluate the adequacy of the formulas to estimate OsmU, the measurements were considered precise if the result was within $30 \mathrm{mOsm} / \mathrm{kg}$ from the measured OsmU, which represents a bounded average of $5 \%$ of the difference between OsmUm and OmsUc with a factor of 35. Differences $>30 \mathrm{mOsm} / \mathrm{kg}$ were considered overestimations, and those $-30 \mathrm{mOsm} / \mathrm{kg}$ were considered underestimations. UD and adjusted UD were both used to calculate the OsmU. For simplicity, urinary samples were classified as clean samples if they did not present proteinuria and glycosuria, and samples with proteinuria and/or glycosuria. The frequency of precise estimations was studied by this classification. 
The results for serum sodium $(\mathrm{Na})$ and serum creatinine $(\mathrm{Cr})$ were taken on the same day that urine samples were collected. The glomerular filtration rate (GFR: $\mathrm{mL} / \mathrm{min} / 1.73 \mathrm{~m}^{2}$ ) was estimated for all patients using the formula CKD-EPI ${ }^{13}$. To compare the behavior of OsmUc in different clinical scenarios, the patients were classified as follows: (1) hyponatremia $(\mathrm{Na}<135 \mathrm{mEq} / \mathrm{L}) ;(2)$ hypernatremia $(\mathrm{Na}>145$ $\mathrm{mEq} / \mathrm{L}$ ); (3) AKI; (4) CKD, according to KDIGO definitions; and (5) other diagnoses ${ }^{13,14}$.

To evaluate the association between OsmUm and both serum sodium concentration and renal function, the patients were classified as having hyponatremia $(\mathrm{Na}<135 \mathrm{mEq} / \mathrm{L})$, hypernatremia $(\mathrm{Na}>145 \mathrm{mEq} / \mathrm{L})$, or normonatremia (135-145 mEq/L). Mild/moderate renal function decrease or greater was defined using the CKD-EPI formula in patients with GFR $<60 \mathrm{~mL}$ / $\mathrm{min} / 1.73 \mathrm{~m}^{2} 14$.

\section{Statistical analysis}

For the quantitative variables following a normal distribution (using Kolmogorov-Smirnov tests to verify the normalcy of the data, with $p<0.05$ meaning to reject the null hypothesis that the data follow a normal distribution), the results were expressed using measures of central tendency (percentual proportions and means) along with their respective measures of dispersion (standard deviation); those that did not present a normal distribution were expressed as proportions, medians, and ranges in percentages. All variables were presented in general and with or without renal injury (AKI or CKD). Differences by renal function were assessed by the Fisher's exact test or Chisquared test, if qualitative, or by Student's $t$-test or Mann-Whitney U-test, if quantitative.

Paired Student's t-test or Wilcoxon signed-ranks tests were applied to assess whether the mean or median of the difference between OsmUm and Os$\mathrm{mUc}$ was zero. This analysis was done with estimations from UD and adjusted UD and by subgroup according to the type of urinary sample (clean or with proteinuria and/or glycosuria). An univariate correlation analysis of the measured OsmU and UD was performed using the Pearson coefficient. Bland-Altman plots were applied to characterize the correlation between OsmUm and OmsUc. Finally, receiver operating characteristic curves were created to illustrate the formulas' diagnostic ability of for the cutoff points $<100$ and $>600 \mathrm{mOsm} / \mathrm{kg}$. The data were processed using the software SPSS version 22.0 (IBM, New York, USA) and the R programming language. $p<0.05$ was considered statistically significant.

\section{RESULTS}

During the study period, 205 samples were collected; $47.3 \%(n=97)$ of the patients were male and $52.7 \%$ $(n=108)$ were female. The median age for the entire study population was 56 years (range 18-95 years). Table 1 shows the population's demographic and clinical characteristics, divided by the presence or absence of renal injury. We observed higher serum osmolarity (OsmS) and more common hypernatremia diagnosis in patients with renal injury (295 vs. 281.8 $\mathrm{mOsm} / \mathrm{kg}, p=0.000 ; 28.8 \%$ vs. $0.62 \%, p=0.000$ ). The correlation between OsmUm and OsmS was not significant $(r=0.120, p=0.086$; Spearman's rank correlation). Hyponatremia was the most frequent diagnosis, representing $45.8 \%$ of the patients; the second most frequent diagnosis was $\mathrm{AKI}$, in $14.6 \%$ of the patients.

Table 2 summarizes the results on the urine parameters and the presence or absence of renalinjury for the total population. Both the UD (including adjusted) and the OsmU (measured and calculated with all factors) were lower in patients with renal injury.

When analyzing the accuracy of the formulas used to estimate OsmU, involving both UD and adjusted UD, factors of 30 and 32 underestimated the $\mathrm{OsmU}$, while 35 and 40 overestimated it. These differences were statistically significant. When using 33.5 , there was no difference between the medians of OsmUm and OsmUc; furthermore, a median of differences from OsmUm was closer to zero with this factor (Fig. 1, Table 3). Furthermore, the highest proportion of accurate estimations (29.8\%) was obtained when using 33.5. When analyzing clean urine samples, factors of 35 and 40 had the highest proportions of accurate estimations ( $31 \%$ and $36.2 \%$, respectively), but there was no difference with OsmUm only when using 35 ( $p=0.844$, Wilcoxon signed-ranks test). For samples with proteinuria and/or glycosuria, the highest proportions of accurate estimations were obtained for the factors $32(30 \%)$ and 33.5 (31.5\%). In addition, 
Table 1. Demographic and clinical characteristics of the study population.

\begin{tabular}{|c|c|c|c|c|}
\hline Variable & Global & No renal injury & Renal injury & $p$ value* \\
\hline$n(\%)$ & $205(100)$ & $160(78)$ & $45(21.9)$ & NA \\
\hline \multirow[t]{2}{*}{ Female sex (\%) } & $108(52.7)$ & $89(55.6)$ & $19(42.2)$ & 0.130 \\
\hline & Median (IQR) & Median (IQR) & Median (IQR) & 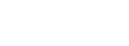 \\
\hline Age (years) & $56(39.5-76)$ & $56(39.25-71)$ & $55(41-68.5)$ & 0.641 \\
\hline \multicolumn{5}{|l|}{ Biochemical parameters } \\
\hline Serum Na (mmol/L) & $135(129-140)$ & 135 (129-139) & $136(127.5-146)$ & 0.000 \\
\hline Serum $\mathrm{Cr}(\mathrm{mg} / \mathrm{dL})$ & $0.86(0.60-1.29)$ & $0.79(0.57-1.00)$ & $1.85(1.30-2.59)$ & 0.000 \\
\hline GFR $\left(\mathrm{mL} / \mathrm{min} / 1.73 \mathrm{~m}^{2}\right)^{\dagger}$ & $81.34 \pm 37.4$ & $91.83 \pm 32.66$ & $44.02 \pm 28.4$ & $* * 0.000$ \\
\hline Serum OsmU (mOsm/kg) & $284.7 \pm 16.8$ & $281.8 \pm 14.6$ & $295.0 \pm 20.1$ & 0.000 \\
\hline Diagnoses & n (\%) & n (\%) & n (\%) & $\frac{\subsetneq}{0}$ \\
\hline Hyponatremia & $94(45.8)$ & $73(45.62)$ & $21(46.6)$ & 1.000 \\
\hline Hypernatremia & $14(6.82)$ & $1(0.62)$ & $13(28.8)$ & 0.000 \\
\hline Acute kidney injury & $30(14.6)$ & & & NA \\
\hline CKD & $15(7.3)$ & & & NA \\
\hline G1-G2 & $1(6.6)$ & & & 3 \\
\hline G3a-G3b & $8(53.3)$ & & & 은 \\
\hline G4-G5 & $6(40)$ & & & (1) \\
\hline
\end{tabular}

${ }^{\dagger}$ GFR: glomerular filtration rate was the only variable with a normal distribution, so it is expressed as mean and standard deviation.

*Kolmogorov-Smirnov test was used for the quantitative variables, while Mann-Whitney U-test was used for the qualitative variables and Fisher's exact test was used to compare dichotomous variables.

**Student's $t$-test. Inner renal injuries were considered acute kidney disease and chronic kidney disease (CKD). The CKD categories were based on the KDIGO 2012 Guidelines ${ }^{14}$. IQR: interquartile range; Na: sodium; Cr: creatinine; GFR: glomerular filtration rate; NA: not applicable.

when using the Wilcoxon signed-ranks test for this type of sample, we found no differences between OsmUm and OsmUc with factors $30(p=0.099), 32$ $(p=0.292), 32$ with adjusted UD $(p=0.898)$, and 33.5 with adjusted UD $(p=0.068)$. The results are shown in figure 2 .

In the linear regression analysis for OsmUm and UD, Pearson's $r=0.788$ and $r^{2}=0.621$, which were statistically significant, with $p<0.001$, so only $62.1 \%$ of the variation in the measured OsmU was explained by the UD. For clean samples, Pearson's $r=0.776$, similar to the one obtained for the total sample. Figure 3 shows the graphic representation of the linear regression.

In the Bland-Altman plots, we observed an increase in the variability of the differences as the magnitude of the measurements increased. This bias is significant because the line of equality is not in the confidence interval $(\mathrm{Cl})$. The Bland-Altman plot for OsmUc with a factor of 35 is shown in figure 4 .
The area under the curve (AUC) obtained by graphing the receiver operating characteristics curves with the different formulas to estimate OsmU value $<100$ $\mathrm{mOsm} / \mathrm{kg}$ was $0.964(95 \% \mathrm{Cl}, 0.906-1.000)$; for OsmU > $600 \mathrm{mOsm} / \mathrm{kg}$, it was 0.907 (95\% Cl, 0.8670.948). For each cutoff point, the same AUC was obtained when using the five formulas.

Table 4 shows the sensitivity (Se), specificity (Sp), positive predictive value, negative predictive value, positive likelihood ratio $(L R+)$, and negative $L R$ - of the five formulas, according to both cutoff points.

The analysis of OsmU in the different groups according to renal function and serum sodium is displayed in table S2. The median for measured OsmU was greater for the group with GFR $\geq 60 \mathrm{~mL} / \mathrm{min} / 1.73 \mathrm{~m}^{2}$ and normonatremia, which constituted $39.5 \%(n=81)$ of the study sample. Measured OsmU was significantly associated with serum sodium $(p=0.002)$ and renal function $(p<0.001)$. 
Table 2. Urinalysis, calculated osmolarity, and measured osmolarity.

\begin{tabular}{|c|c|c|c|c|}
\hline \multirow[t]{2}{*}{ Variable } & \multicolumn{3}{|c|}{ Median (IQR) } & \multirow{2}{*}{$p$ value } \\
\hline & Global & No renal injury & Renal injury & \\
\hline$n(\%)$ & $205(100)$ & $160(78)$ & $45(21.9)$ & NA \\
\hline Urinary density & $1.014(1.009-1.018)$ & $1.014(1.009-1.018)$ & $1.011(1.006-1.018)$ & 0.030 \\
\hline Adjusted density & $1.014(1.009-1.018)$ & $1.014(1.009-1.018)$ & $1.011(1.005-1.017)$ & 0.021 \\
\hline \multirow[t]{2}{*}{ Urinary $\mathrm{pH}$} & $6(5-7)$ & $6(5-7)$ & $5(5-7)$ & 0.831 \\
\hline & $\mathrm{n}(\%)$ & $\mathrm{n}(\%)$ & $\mathrm{n}(\%)$ & $\frac{\bar{n}}{0}$ \\
\hline Proteins & & & & 0.169 \\
\hline Negative & $137(66.8)$ & $113(70.6)$ & $24(53.3)$ & $\stackrel{\oplus}{\risingdotseq}$ \\
\hline $1(+)$ & $33(16.1)$ & $22(13.8)$ & $11(24.4)$ & 4 \\
\hline $2(++)$ & $27(13.2)$ & $19(11.9)$ & $8(17.8)$ & $\frac{0}{n}$ \\
\hline $3(+++)$ & $8(3.9)$ & $6(3.8)$ & $2(4.4)$ & $\frac{\omega}{\varepsilon}$ \\
\hline Glucose & & & & 0.536 \\
\hline Negative & $164(80.0)$ & $130(81.3)$ & $34(75.6)$ & $\frac{\varsigma}{d}$ \\
\hline $1(+)$ & $28(13.7)$ & $19(11.9)$ & $9(20.0)$ & $\frac{ \pm}{5}$ \\
\hline $2(++)$ & $7(3.4)$ & $6(3.8)$ & $1(2.2)$ & - \\
\hline $3(+++)$ & $6(2.9)$ & $5(3.1)$ & $1(2.2)$ & 흘 \\
\hline Ketones & & & & 0.798 \\
\hline Negative & $174(84.9)$ & $136(85.0)$ & $38(84.4)$ & 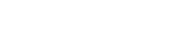 \\
\hline Traces & $18(8.8)$ & $13(8.1)$ & $5(11.1)$ & $\stackrel{f}{\rightleftharpoons}$ \\
\hline $1(+)$ & $11(5.4)$ & $9(5.6)$ & $2(4.4)$ & 3 \\
\hline $2(++)$ & $2(1.0)$ & $2(1.3)$ & $0(0.0)$ & 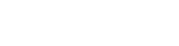 \\
\hline Bilirubin & & & & 0.035 \\
\hline Negative & $196(95.6)$ & $155(96.9)$ & $41(91.1)$ & $\stackrel{t}{\circ}$ \\
\hline $1(+)$ & $5(2.4)$ & $4(2.5)$ & $1(2.2)$ & 은 \\
\hline $2(++)$ & $4(2.0)$ & $1(0.6)$ & $3(6.7)$ & $\frac{0}{0}$ \\
\hline Hemoglobin & & & & $<0.001$ \\
\hline Negative & $105(51.2)$ & $94(58.8)$ & $11(24.4)$ & o \\
\hline $1(+)$ & $58(28.3)$ & $44(27.5)$ & $14(31.1)$ & 인 \\
\hline $2(++)$ & $29(14.1)$ & $16(10.0)$ & $13(28.9)$ & 일 \\
\hline $3(+++)$ & $13(6.3)$ & $6(3.8)$ & $7(15.6)$ & है \\
\hline \multirow[t]{2}{*}{ Clean samples ${ }^{\dagger}$} & $116(56.6)$ & $95(59.4)$ & $21(46.7)$ & 0.173 \\
\hline & Median (IQR) & Median (IQR) & Median (IQR) & Uु \\
\hline \multicolumn{5}{|l|}{ OsmU (mOsm/kg) } \\
\hline Measured OsmU & $425(309-582)$ & $473(350-608)$ & $320(238-447)$ & $<0.001$ \\
\hline Calculated OsmU ×30 & $420(270-540)$ & $420(270-562)$ & $330(180-540)$ & 0.030 \\
\hline Calculated OsmU ×32 & $448(288-576)$ & $448(288-600)$ & $352(192-756)$ & 0.030 \\
\hline Calculated OsmU ×33.5 & $469(301-603)$ & $469(301-628)$ & $368(201-603)$ & 0.030 \\
\hline Calculated OsmU ×35 & $490(315-630)$ & $490(315-656)$ & $385(210-630)$ & 0.030 \\
\hline Calculated OsmU ×40 & $560(360-720)$ & $560(360-750)$ & $440(240-720)$ & 0.030 \\
\hline
\end{tabular}

${ }^{\dagger}$ Clean samples were those without glycosuria or proteinuria.

*Mann-Whitney U-test was used to compare quantitative variables, while Fisher's exact test was used for clean samples variable and $X^{2}$ was used for the rest. NA: not applicable; IQR: interquartile range; OsmU: urinary osmolarity 
Figure 1. The differences between measured and calculated osmolarity using the five formulas OsmU, urinary osmolarity; m, measured by osmometer; c, calculated by urinary density.

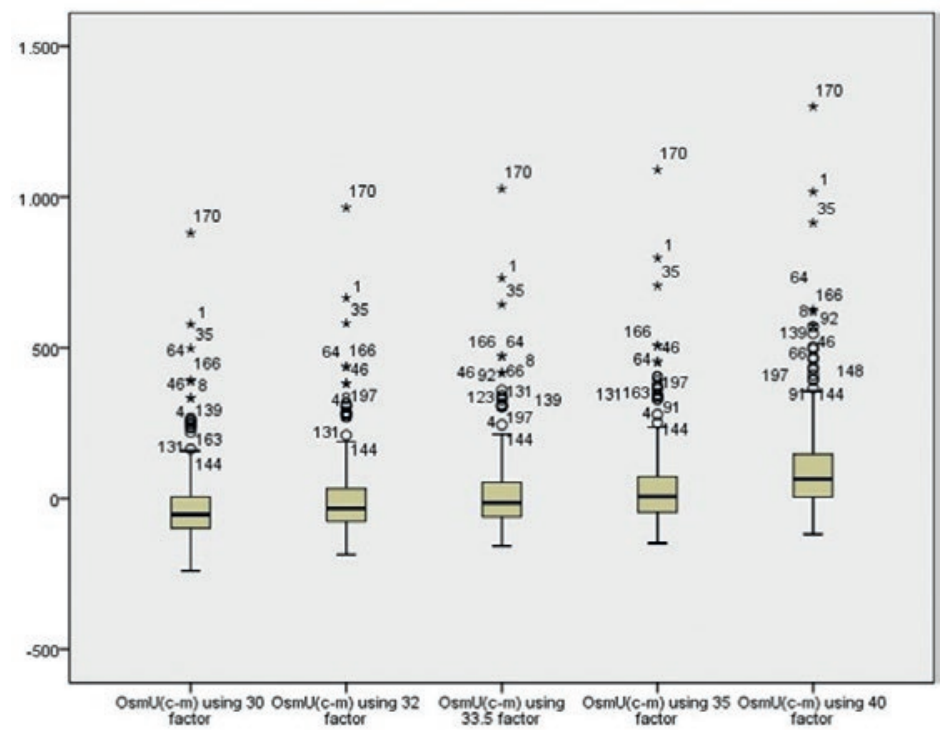

Figure 2. Urinary osmolarity estimations by formula and type of urine sample (clean or with proteinuria and/or glycosuria). Differences $>30 \mathrm{mOsm} / \mathrm{kg}$ were considered overestimations, and differences lower than $-30 \mathrm{mOsm} / \mathrm{kg}$ were considered underestimations. P-uria, proteinuria; G-uria, glycosuria.

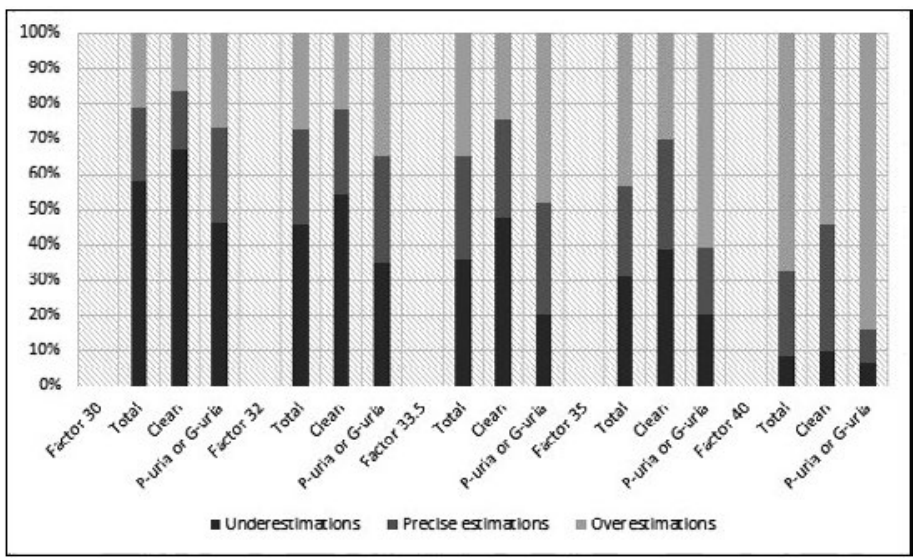

\section{DISCUSSION}

Historically, diverse methods have been used to estimate the OsmU from the UD. Nonetheless, it is controversial that OsmUc and OsmUm are adequately correlated, and very few studies about this correlation exist. The first published studies showed high degrees of correlation $(r=0.93-0.99)^{15,16}$; however, a recent work on this topic showed a low correlation between both parameters $(r=0.462, p<0.0001)^{10}$. In the present study, a high, positive, statistically significant linear correlation was found between
OsmUm and UD ( $\left.r=0.73-0.86, r^{2}=0.621\right)$, in the middle of the previously reported values; thus, $62.1 \%$ of the variation in the measured OsmU was explained by the UD. UD measurement by refractometry or by reactive strips involves multiple factors such as the presence of proteinuria, glycosuria, contrast agents, mannitol, ketonuria, bilirubin, urobilinogen, and hemoglobinuria as well as $\mathrm{pH}$, which may intervene with the results $13,7,11,17,18$. This could explain the variability between the correlation coefficients reported by different authors. For example, some studies described a better correlation when 
Figure 3. Dispersion diagram of measured OsmU versus urinary density. OsmU, urinary osmolarity.

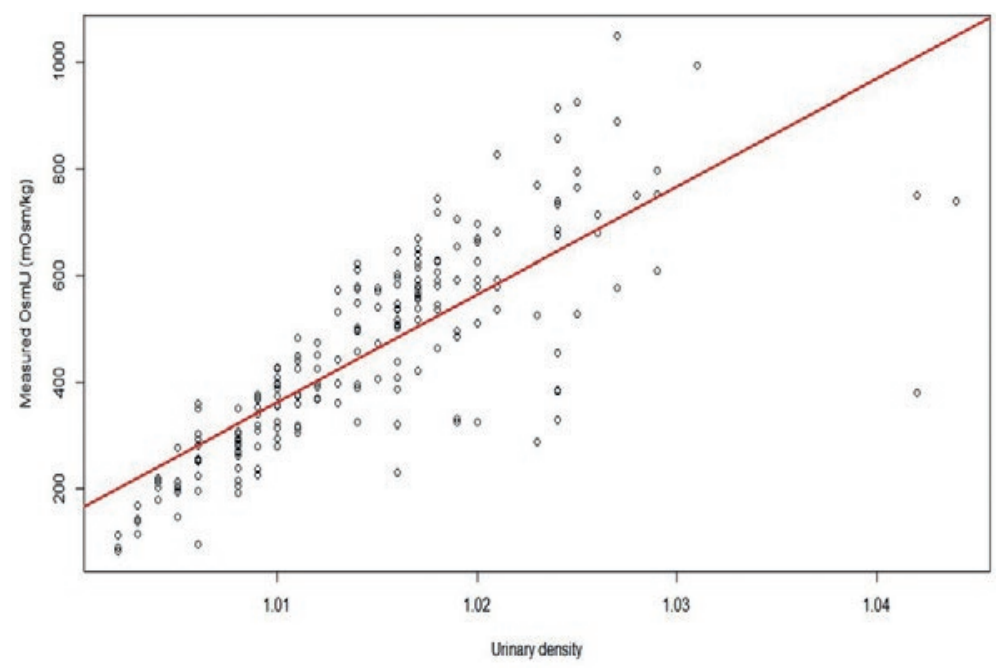

Figure 4. Bland-Altman plot for the OsmUc with factor of $\times 35$. The dashed lines represent the $95 \%$ confidence interval of the mean. OsmUm, measured urinary osmolarity; OsmUc, calculated urinary osmolarity.

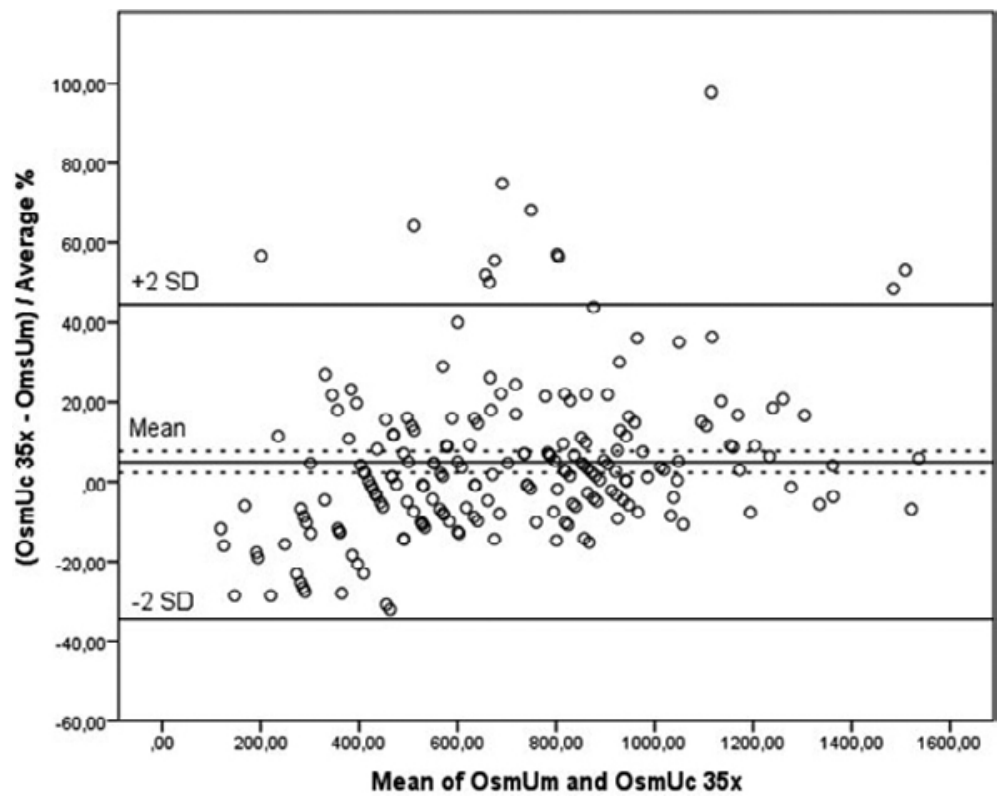

analyzing clean samples $(r=0.572-0.83)$ than samples with proteinuria and/or glycosuria ( $r=0.459$ $0.66)^{10,18}$. Some authors have proposed corrections to lessen the impact of these solutes in determining the OsmU from the DU, although not every study has found a better correlation with such adjustments, probably due to the low prevalence of altered urinary samples ${ }^{1,2}$.

In the present study, a better correlation with OsmU was not found when evaluating UD adjusted for glycosuria and proteinuria $(r=0.776)$. This could be explained, to some extent, by the very low frequency of samples with significant glycosuria and proteinuria $(3+)$, which represented $2.9 \%$ and $3.9 \%$ of the cases, respectively. On the other hand, the amounts of other solutes found normally in the urine, such as sodium, potassium, and urea, and of exogenous solutes that are not routinely detected in urinary samples (e.g., drugs and contrast agents for imaging studies) may modify the UD, thus altering the correlation between UD and OsmUm ${ }^{18}$. 
Table 3. Difference between calculated OsmU (OsmUc) and calculated adjusted OsmU (OsmUca) medians with measured OsmU (OsmUm).

\begin{tabular}{|c|c|c|c|c|c|}
\hline \multirow[t]{2}{*}{ Formula $^{\dagger}$} & Factor $\times 30$ & Factor $\times 32$ & Factor $\times 33.5$ & Factor $\times 35$ & Factor $\times 40$ \\
\hline & Median (IQR) & Median (IQR) & Median (IQR) & Median (IQR) & Median (IQR) \\
\hline OsmUm & $425(309,582)$ & $425(309,582)$ & $425(309,582)$ & $425(309,582)$ & $425(309,582)$ \\
\hline OsmUc & $420(270,540)$ & $448(288,576)$ & $469(301.5,603)$ & $490(315,630)$ & $560(360,720)$ \\
\hline OsmUca & $420(270,540)$ & $448(288,576)$ & $469(301.5,603)$ & $490(315,630)$ & $560(360,720)$ \\
\hline OsmUc - OsmUm & $-48(-95,9.5)$ & $-24(-72.5,39.0)$ & $-4.5(-55.2,63.2)$ & $+12(-41.5,82.5)$ & $+81(8.5,163.5)$ \\
\hline$p$ value* & $<0.001$ & 0.026 & 0.578 & 0.001 & $<0.001$ \\
\hline OsmUca - OsmUm & $-53.0(-98,7.1)$ & $-32.6(-75.1,-34.0)$ & $-13.6(-60,55.7)$ & $+6.0(-45.0,73.6)$ & $+65.0(5.0,149.5)$ \\
\hline$p$ value* & $<0.001$ & 0.003 & 0.695 & 0.016 & $<0.001$ \\
\hline
\end{tabular}

${ }^{\dagger} \mathrm{OmsU}$ is expressed in $\mathrm{mOsm} / \mathrm{kg}$.

* $p$ values using the Wilcoxon signed-rank test.

Comparison between OsmUm and OsmUc or OsmUca from each factor. OsmU: urinary osmolarity; IQR: interquartile range.

Table 4. Internal and external validity for the different cutoff points of OsmU.

\begin{tabular}{|c|c|c|c|c|c|c|c|}
\hline Formula & Se $(\%)$ & Sp (\%) & PPV (\%) & NPV (\%) & LR+ & LR- & Youden index \\
\hline \multicolumn{7}{|c|}{ To detect OsmU $<100 \mathrm{mOsm} / \mathrm{kg}$} & $\frac{1}{2}$ \\
\hline Factor $\times 30$ & 66.7 & 97.5 & 27.5 & 99.5 & 26.6 & 0.342 & 0.642 \\
\hline Factor $\times 32$ & 66.7 & 97.5 & 27.5 & 99.5 & 26.6 & 0.342 & 0.642 \\
\hline Factor $\times 33.5$ & 66.7 & 99.5 & 65.4 & 99.5 & 113.4 & 0.335 & 0.662 \\
\hline Factor $\times 35$ & 66.7 & 99.5 & 65.4 & 99.5 & 113.4 & 0.335 & 0.662 \\
\hline Factor $\times 40$ & 66.7 & 99.5 & 65.4 & 99.5 & 113.4 & 0.335 & 0.662 \\
\hline \multicolumn{7}{|c|}{ To detect OsmU > $600 \mathrm{mOsm} / \mathrm{kg}$} & $\frac{0}{0}$ \\
\hline Factor $\times 30$ & 53.5 & 92.6 & 65.6 & 88.3 & 7.2 & 0.502 & 0.461 \\
\hline Factor $\times 32$ & 67.4 & 87.0 & 57.8 & 91.0 & 5.1 & 0.375 & 0.544 \\
\hline Factor $\times 33.5$ & 79.1 & 84.0 & 56.6 & 93.8 & 4.9 & 0.249 & 0.631 \\
\hline Factor $\times 35$ & 79.1 & 84.0 & 56.6 & 93.8 & 4.9 & 0.249 & 0.631 \\
\hline Factor $\times 40$ & 95.3 & 69.8 & 45.5 & 98.3 & 3.1 & 0.067 & 0.651 \\
\hline
\end{tabular}

OsmU: urinary osmolarity; Se: sensitivity; Sp: specificity; PPV: positive predictive value; NPP: negative predictive value; LR+: positive likelihood ratio; LR-: negative likelihood ratio.

When evaluating the accuracy of the different formulas described for the indirect estimation of OsmU from UD, no difference with OsmUm was found when using a factor of 33.5. Interestingly, when evaluating merely clean samples, no difference with OsmUm existed only when using a factor of 35 . On the other hand, when analyzing samples with proteinuria and/ or glycosuria, a factor of 32 with adjusted UD had the closest proximity to the OsmUm. As far as we know, this finding has not been previously reported and opens the possibility of evaluating the use of different factors according to the characteristics of the urine and the clinical scenario.
The evaluation of the five formulas for OsmU estimation in samples with OsmU $<100$ and $>600$ mOsm/ $\mathrm{kg}$ showed that they all may provide suitable diagnostic discrimination ( $A \cup C>0.90$ ). Nevertheless, very few samples had OsmU < $100 \mathrm{mOsm} / \mathrm{kg}$; therefore, the results may not be definitive in this range. That consideration is transcendent, even though the specificity is near $100 \%$ because these values are used in routine clinical practice to assess patients presenting with hyponatremia and polyuria, especially in cases with diabetes insipidus ${ }^{19}$. In addition, we observed greater variability at higher magnitudes of measurement. Therefore, clinical judgment should be applied when 
making treatment decisions using any of the formulas to calculate the OsmU from the DU.

We observed that urinary concentration capacity was compromised in patients with deteriorated renal function, resulting in lower OsmU, and worse renal function. Hence, serum sodium concentration and renal function are significantly related to OsmUm, thus confirming the physiological basis by which patients with normal renal function have more variable OsmU based on extracellular volume and plasmatic osmolarity regulating factors.

Among the limitations to this study are the lower proportions of patients included in groups other than the hyponatremia group. Furthermore, we were not able to evaluate the influence of other causes that can affect the assessment of OsmU, including medications ${ }^{10}$.

Regarding the strengths of the work, it is important to highlight that almost $45 \%$ from the total study population (92 patients) had hyponatremia. This is relevant because one of the most frequent uses of OsmU in clinical practice is for determining the approach to electrolytic disorders, although to the best of our knowledge, no works have been published related to the estimation of OsmU from UD in these patients. The correlation analysis between OsmUm by osmometry and its estimation from the UD found during UA with the factors (multiply by $30,32,33.5$, 35 , and 40 ) used in clinical practice is pertinent because previous studies have focused only on analyzing UD as a direct numerical value against OsmUm. Finally, relevant cutoff points for OsmU were analyzed regarding the approach to pathology, for which the evaluation of urinary concentration capacity is fundamental. In general, factors of 30 and 32 underestimated the OsmU values, while factors of 35 and 40 overestimated the measurement. With OsmU values close to $100 \mathrm{mOsm} / \mathrm{kg}$, we recommend corroborate the results of direct measurement by osmometry, due to the low sensitivity of the calculated form. Glycosuria and proteinuria alter the OsmUc results, so we recommend using a factor of 32 with adjusted DU for samples with proteinuria and/or glycosuria but 35 for clean samples. When the characteristics of the UA are unknown or unreliable, it seems better to use 33.5 as the factor. Other causes influencing the measured OsmU should be analyzed in subsequent studies.

The estimation of the OsmU from DU is acceptable. However, several agents alter the accuracy of the estimate, so we recommend measuring OsmU by osmometry, as long as it is available, to evaluate the urinary concentration capacity in clinical practice.

\section{SUPPLEMENTARY DATA}

Supplementary data are available at Revista de Investigación Clínica online (www.clinicalandtranslationalinvestigation.com). These data are provided by the corresponding author and published online for the benefit of the reader. The contents of supplementary data are the sole responsibility of the authors.

\section{REFERENCES}

1. Chadha V, Garg U, Alon US. Measurement of urinary concentration: a critical appraisal of methodologies. Pediatr Nephrol. 2001;16:374-82.

2. Rose BD, Post TW, editors. Clinical Physiology of Acid Base and Electrolyte Disorders. 5th ed. New York: Mc Graw Hill; 2001.

3. Elias-Costa C, Bettendorff MC, Bupo S, Ayuso S, Vallejo G. Medición comparativa de la densidad urinaria: tira reactiva, refractómetro y densitómetro. Arch Argent Pediatr. 2010;108:234-8.

4. List AF, Hainsworh JD, Davis BW, Hande KR, Greco FA, Johnson $\mathrm{DH}$. The syndrome of inappropriate secretion of antidiuretic hormone (SIADH) in small-cell lung cancer. J Clin Oncol. 1986;4:1191-8.

5. Spasovski G, Vanholder R, Allolio B, et al. Clinical practice guideline on diagnosis and treatment of hyponatraemia. Nephrol Dial Transplant. 2014;29 Suppl 2:11-139.

6. Hoorn EJ, Halperin ML, Zietse R. Diagnostic approach to a patient with hiponatremia: traditional versus physiology based options. QMJ. 2005;98:529-40.

7. Perrier ET, Bottin JH, Vecchio M, Lemetais G. Criterion values for urine-specific gravity and urine color representing adequate water intake in healthy adults. Eur J Clin Nutr. 2017;71:561-3.

8. Yeh HC, Lin YS, Kuo CC, et al. Urine osmolality in the US population: implications for environmental biomonitoring. Environ Res. 2015;136:482-90.

9. Voinescu G, Shoemaker M, Moore H, Khanna R, Nolph KD. The relationship between osmolality and specific gravity. Am J Med Sci. 2002;323:39-42.

10. Souza AC, Zatz R, de Oliveira RB, et al. Is urinary density an adequate predictor of urinary osmolality? BMC Nephrol.2015;16:46.

11. Miller BE, Paton A, de Wardener HE. Maximum urine concentration. Br Med J. 1954;2:901-5.

12. Miller RB. Urinalysis. In: Massry SG, Glassock RJ, editors. Textbook of Nephrology. Baltimore: Williams and Wilkins; 1983. p. 11.3-24.

13. Improving Global Outcomes (KDIGO) Acute Kidney Injury Work Group. KDIGO clinical practice guideline for acute kidney injury. Kidney Inter. 2012;2:1-138.

14. Improving Global Outcomes (KDIGO) CKD Work Group. KDIGO 2012 clinical practice guideline for the evaluation and management of chronic kidney disease. Kidney Inter Suppl. 2013;3:1-150.

15. Bakhshandeh S, Morita Y. Comparison of urinary concentration tests: osmolality, specific gravity, and refractive index. Mich Med. 1975;74:399-403.

16. Dorizzi R, Pradella M, Bertoldo S, Rigolin F. Refractometry, test strip, and osmometry compared as measures of relative density of urine. Clin Chem. 1987;33:190.

17. Medler S, Harrington F. Measuring dynamic kidney function in an undergraduate physiology laboratory. Adv Physiol Educ. 2013;37:384-91.

18. Imran S, Eva G, Christopher S, Flynn E, Henner D. Is specific gravity a good estimate of urine osmolality? J Clin Lab Anal. 2010; 24:426-30.

19. Lamas C, De Pozo C, Villabona C. Guía clínica de manejo de la diabetes insípida y del síndrome de secreción inapropiada de hormona antidiurética en el postoperatorio de la cirugía hipofisaria. Endocrinol Nutr. 2014;61:e12-5. 